\title{
UNDERSTANDING LIFE PROCESS BY CHEMISTRY - A Tribute to the Contributions of Professor Yuichi Kanaoka in Heterocyclic Chemistry and Bioorganic Chemistry -
}

It is our great pleasure and honor to introduce the accomplishments of Professor Kanaoka's research in this special issue of Heterocycles on the occasion of his 75 th birthday. Professor Kanaoka made an enormous contribution as a Japanese pioneer not only in nitrogen-heterocyclic chemistry, but also in the wide field of bioorganic chemistry, the interface between chemistry and biology. His achievements that were generated by his far-sighted discernment for the future of science will be remembered long into the 21 st century. In the following, the contributions of Professor Kanaoka are briefly summarized.

Professor Kanaoka began his scientific career at the University of Tokyo with the late Professor Shigehiko Sugasawa, studying an application of the Robinson dehydrogenation to heterocyclic synthesis. After moving to Hokkaido University in 1956, he initiated research in bioorganic chemistry by applying polyphosphate esters to synthesize various compounds of biological importance.

\section{Synthetic Application of Polyphosphate Esters (PPE) to Various Compounds of Biological Importance}

PPE, a Lewis-type acid, less protic and more lipophilic than polyphosphoric acid, acts as a mild dehydrating agent in organic solvents. In addition to the ability to activate carboxylic acids and acid amides, the reagent was used for rearrangement and alkylation in Fischer's indole synthesis. The compounds synthesized include benazepines, indoles, tryptamine and tryptophan derivatives, and peptides. Benzimidazoles and amidines were also synthesized, and were to be revisited in his later research.

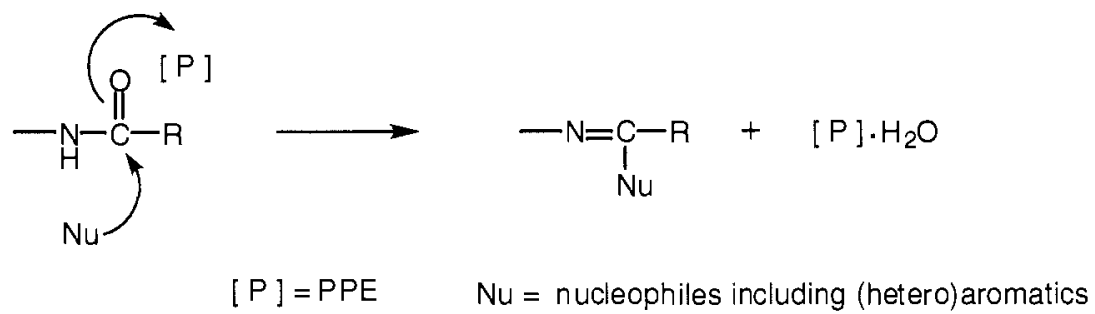

Professor Kanaoka's wish to invade and develop the research in bioorganic chemistry was inspired during his stay at Dr. Bernhard Witkop's laboratory at NIH, as a Visiting Associate (1959-1961). He often talked us how exciting and impressive days he spent there, in the worldwide "melting pot" of scientific 
disciplines centering on medical research that obviously needed the participation of chemistry. The close relationship between Dr. Witkop and Professor Kanaoka, as a respected mentor and as a younger colleague and "Japanese-teaching" tutor, respectively, has lasted through their long scientific careers.

Massive works started soon after Professor Kanaoka returned to Hokkaido University. He initially focused on two subjects: developing ultra-sensitive chemicals for monitoring biological systems and "chemical knives" designed to reveal the anatomy of enzyme-catalyzed mechanisms.

\section{Development of Fluorescent Reagents: Ultra-sensitive Monitors for Biomolecules}

Thiols, the ubiquitous and chemically most reactive groups found in cells, were selected as a special target. 2-Phenylbenzimidazole was selected as a fluorophore that could be conjugated with maleimide as a thiol-specific functional group. Unexpectedly, the maleimide reagent itself was not fluorescent, but became highly fluorescent after reaction with thiols. The intriguing phenomenon was explained as follows: The excited singlet $\left(n, \pi^{*}\right)$ state of maleimide-type reagent can efficiently proceed through intersystem crossing to the triplet state and hence is not fluorescent, while the crossing is prohibited for the higher excited singlet state of the succinimide-type product to resulting in high fluorescence. A series of maleimide-type reagents were developed with different fluorescent characteristics as follows:

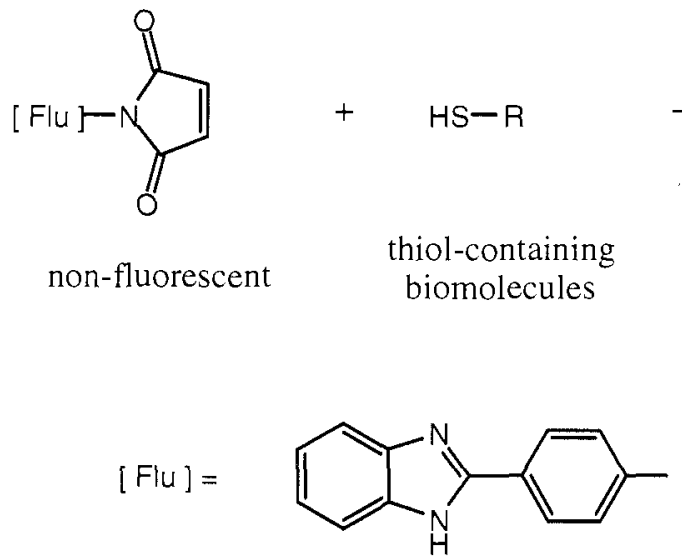

(BIPM)

$(\mathrm{ANM})$<smiles>Cc1ccc(Nc2ccccc2)c2ccccc12</smiles><smiles>[2H]SC1CC(=O)N([AlH2])C1=O</smiles>

highly fluorescent<smiles>Cc1c(C)c2ccc(N(C)C)cc2oc1=O</smiles>

(DACM)<smiles>Cc1ccc2c3c(cccc13)-c1ccccc1-2</smiles>

(FAM) etc. 
the pilotage compound with moderate flurorescent properties (BIPM); fluorescence wavelength (DACM); hydrophobic sensitivity to surrounded microenvironment (ANM); life time of fluorescence (FAM). Such maleimides are called "Kanaoka-Sekine-Machida reagents" to acknowledge the three collaborators. A number of further collaborations were achieved to study localization of thiol-containing biomolecules, depending upon space and time, in living cells and tissues using these "sensors". In addition, 4-methyl-7aminocoumarine-containing fluorogenic substrates (see below) were proposed as sensitive detecting tools for various enzymes of clinical and diagnostic importance. These maleimide reagents and peptide substrates are commercially available and continue to be used as important probes. The perspective of these fluorescent reagents was extensively reviewed by Professor Kanaoka, as invited by Angewandte Chemie in 1977.<smiles>[R]C(=O)Nc1ccc2c(C)cc(=O)oc2c1</smiles>

\section{Synthetic Substrates and Inhibitors for Enzymes based on a Novel Concept}

The second target compounds were the "chemical knives", designed to reveal enzyme structures and functions. Amidinophenyl compounds showed specific binding to trypsin and trypsin-type proteases, mainly due to the positive charge of the amidine group. Therefore, they served a series of tailor-made substrates and inhibitors for the enzymes. $p$-Nitrophenyl ester of $p$-amidinobenzoic acid was hydrolyzed by trypsin and trypsin-type proteases with rapid acylation followed by slow deacylation, providing a useful titrant for active enzyme determination. By contrast, p-amidinophenyl esters of appropriate carboxylic acids, in which the positive charge lay on the leaving phenol moiety, showed rapid acylation, but essentially no deacylation by trypsin. Such novel substrates, which were named "inverse substrates", made it possible to specifically prepare and isolate a variety of stable acyl-enzyme intermediates.

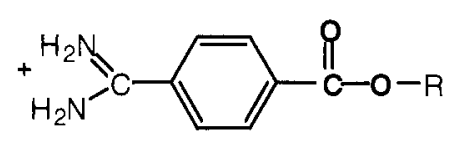

normal-type substrates

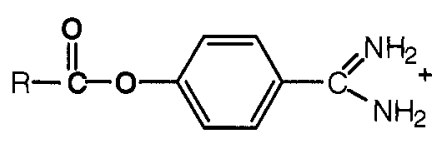

"inverse substrates"

First, compounds with various acyl groups, which included a chromogenic pair of enantiomeric amino acids, fluorescent moieties, and spin-labels as reporters, were synthesized and used to provide dynamic information about microenvironments of the active site during enzyme catalysis. Second, this concept provided a new methodology to prepare acyl-enzymes as enzyme precursors with desired lifetime for 
potential clinical use. The results were reviewed in Accounts of Chemical Research in 1987 by Professor Kanaoka and his colleagues.

\section{Organic Photochemistry of Nitrogen-Conjugated Carbonyl Systems}

After remarkable success in developing unique fluorescent reagents, Professor Kanaoka's scientific interest in the frontier of excited-state chemistry moved to carbonyl photochemistry. In 1970's the research interest of organic photo-chemists was focused on the photochemistry of simple carbonyl system. The research of Professor Kanaoka and his group on the photoreaction of imides and amides uncovered the unique and important excited-state nature of nitrogen-conjugated carbonyl systems. He showed, for the first time, the synthetic utility of these photo-sensitive systems for the construction of various new nitrogen-heterocycles.

Many complex heterocyclic frameworks, including macrocycles, were synthesized from simple photoreaction of readily prepared nitrogen-conjugated carbonyl systems. This photochemical field that Professor Kanaoka developed is still growing and expanding to include sulfur-containing imide systems, readily prepared nitrogen-conjugated carbonyl systems. These results were reviewed by Professor Kanaoka in Accounts of Chemical Research in 1978.

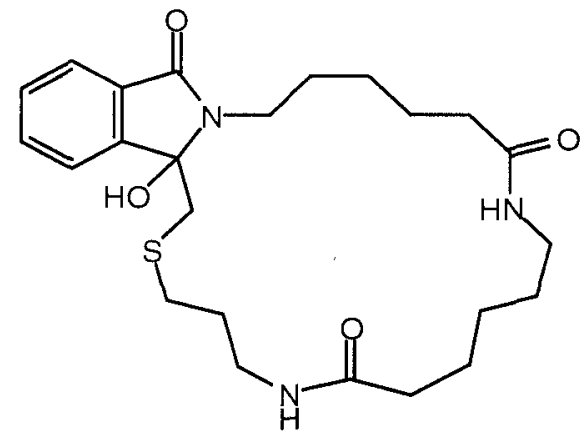

\section{Chemical Understanding of Ion Channels}

In 1980s Professor Kanaoka's research interests moved into the field of neuroscience.

As the initial work, his group focused on the challenge of elucidating the structure of the voltage-sensitive sodium channel that plays a major role for nerve excitation. Through the use of radiolabeled tetrodotoxin (TTX) binding as an assay, a $\mathrm{Na}^{+}$channel protein of $260 \mathrm{kDa}$ was finally obtained in pure form, and tryptic fragments isolated from it were subjected to the microsequence analysis in collaboration with Professor Hisayuki Matsuo. The amino acid sequences obtained were utilized for the molecular cloning of cDNA and the subsequent total sequence deduction. Such studies were collaboratively achieved in the laboratory of late Professor Shosaku Numa. They represented an inaugurating work with respect to revealing the chemical structure of an ion channel and led to the now well-known transmembrane model of 6 segments $x 4$ domains. Professor Kanaoka's group also characterized the tetrodotoxin-binding site by 


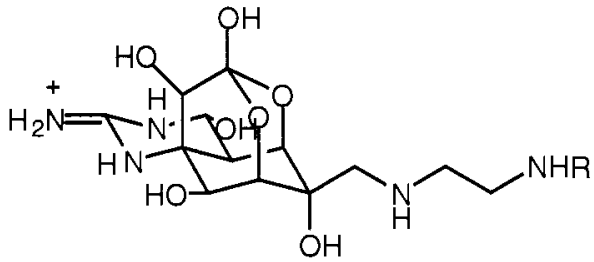

TTX analog<smiles>CCOCOC1=C(C)NC(C)=C(C(=O)OCCNP)C1C(C)(C)C(F)(F)F</smiles>

DHP analog<smiles>[B]=C(C)c1ccc(C2(C(F)(F)F)N=N2)cc1</smiles>

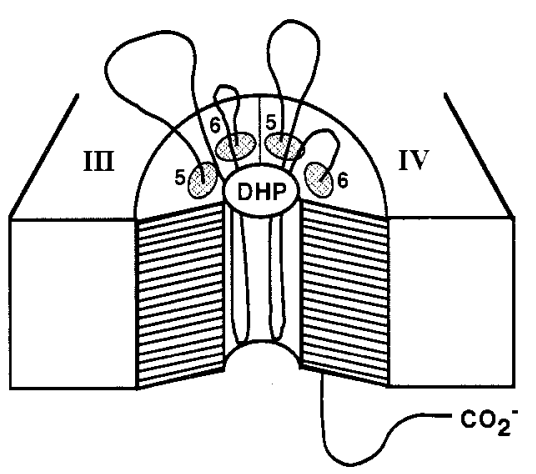

DHP binding site in the $\alpha$ 1 subunit of $\mathrm{Ca}^{2+}$ channel identified by photoaffinity labeling.

photoaffinity-labeling. The results were consistent with those obtained by the site-directed mutagenesis method.

Another example of an elucidation of ion channel structure by Professor Kanaoka's group was the chemical identification of drug-binding sites in L-type $\mathrm{Ca}^{2+}$ channel. 1,4-Dihydropyridine (DHP) is one of the most popular $\mathrm{Ca}^{2+}$ antagonists used clinically as anti-hypertensive agents. The binding site for DHPs was identified by the photoaffinity labeling technique (as shown above). In the history of medicines, it is very rare that "a real receptor for a synthetic drug in vivo" is elucidated at a molecular level. The results stimulated further investigations of the L-type Calcium channels by site-directed mutagenesis. Binding sites for other $\mathrm{Ca}^{2+}$ antagonists that allosterically interact one another, have now been investigated.

\section{Photoaffinity Labeling: A Versatile Method to Reveal Target Molecules}

To make the photoaffinity labeling technique applicable more generally and easily for a variety of biological systems, Professor Kanaoka and his colleagues have made continuous efforts to develop novel, new photosensitive probes. (Trifluoromethyl)phenyldiazirine (TPD) was used as the key photosensitive group, since it generates on irradiation a short-lived phenylcarbene that forms stable photoproducts with relatively high incorporation efficiency. TPD derivatives having different functional groups were proposed as a set of versatile photolabeling reagents. For example, introduction of a phenolic $\mathrm{OH}$ and/or carboxyl group into TPD provides scaffold(s) to connect with ligand molecules. Conjugation with ligand through the carboxyl group and with biotin through the phenolic hydroxyl group enabled photolabeled target molecules to be selectively isolated using the sophisticated avidin-biotin system. Reviews by 


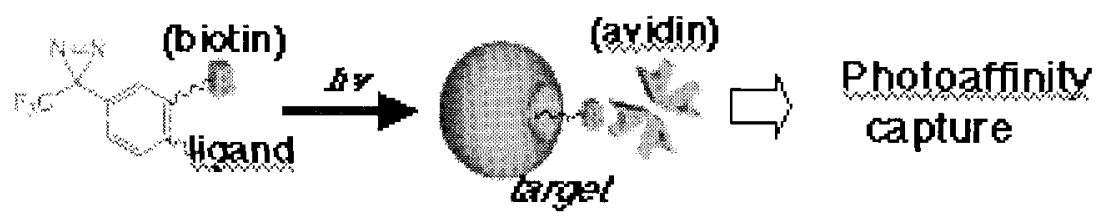

Kanaoka's group appeared in Reviews of Heteroatom Chemistry in 1996 and in Heterocycles in 1998. The elegant method continues to be used successfully by other researchers, including his former coworkers.

\section{Towards Advancement of Science}

Professor Kanaoka participated in the first U.S.-Japan Joint Meeting for Bioorganic Chemistry, Honolulu, 1975, organized by Professors Teruaki Mukaiyama and Ronald Breslow. Many famous chemists, including Professors D. Cram, J.R. Knowles, JP. Collman, Z. Yoshida, the late T. Kaiser, the late T. Kametani, and the late I. Tabushi, attended this meeting.

In 1980s Ministry of Education, Science and Culture of Japan, raised Grant-in-Aid for Scientific Research on Priority Areas. In collaboration with late Professor Yoshio Ban, Professor Kanaoka organized "Nitrogen Organic Resources", the first project in organic chemistry. Successively he raised the special project "Syntheses to Reveal Bio-functions" (1986-1988) to promote research in bioorganic chemistry.

Professor Kanaoka was President of the Pharmaceutical Society of Japan (1992-1994) , and served to enhance activities of that Society of the more than twenty thousand members. It is worth noting that two symposia he initially organized for the society are still continuing, namely "Reactions and Syntheses (27th in 2001)" and "Medicinal Chemistry (21st in 2001)". The French-Japanese Symposium for Medicinal and Fine Chemistry was initiated in 1981 by Professors Pierre Potier and the late Yoshio Ban and was continued by Professors Kanaoka and Osamu Yonemitsu. It has now a history of twenty years.

The Science Council of Japan (SCJ, 210 members) was the representative organization of Japanese scientists, attached to the Prime Minister's Office. Professor Kanaoka was elected to a member as recommended from the Pharmaceutical Society of Japan. From 1997 to 2000 he served as Chairman of the 7th Division (33 members) of SCJ, which covers Medicine, Dentistry, and Pharmaceutical Sciences. It was for the first time that Chairman was elected from the pharmaceutical area during the half-century history of SCJ. He is now Vice-President of Japan Academy of Medical, Dental, and Pharmaceutical Sciences, in support of the 7 th Division. 
"Molecular recognition in life process" is the keyword throughout Professor Kanaoka's research. The concept of molecular recognition is considered by many to have originated from the German chemists, E. Fisher and P. Ehrlich. In modern science, that legacy has been followed by many, including Drs. Bernhard Witkop and his protege Dr. John Daly at NIH, with whom Professor Kanaoka spent three years early in his career. The concept of molecular (chemical) recognition obviously underlies the recent focus on the role of chemistry in the "life sciences". Current "Chemical Biology" initiatives reminds us again of scientific discernment of Professor Kanaoka, who taught us the importance of the concept, and sought it in every aspect of his research. He is now the President of Toyama International Institute, and the associated university and college as well. There is no doubt that his far-sightedness with global impact of his research will strongly affect the young generations, who shoulder the responsibility in this 21 st century.

Minoru MACHIDA, Kazutaka TANIZAWA, Eisuke SATO, Hitoshi NAKAYAMA, and Yasumaru HATANAKA 